\title{
COMMON FIXED POINTS FOR COMMUTING AND COMPATIBLE MAPS ON COMPACTA
}

\author{
GERALD JUNGCK
}

(Communicated by James E. West)

\begin{abstract}
Compatible maps-a generalization of commuting maps-are characterized in terms of coincidence points, and common fixed point theorems for compatible maps and commuting maps on compact metric spaces are obtained.
\end{abstract}

1. Introduction. Maps $f, g: X \rightarrow X$ are said to commute iff $f g=g f$. The concept of commuting maps has proven useful for generalizing in the context of metric space fixed point theory (see, e.g., $[1,2,4-11,15,16,17])$. Recently a less restrictive concept called compatibility was introduced in [12] and promoted as a means to more comprehensive results.

Now any two self-maps $f$ and $g$ of a set $X$ commute on the set $\{x \in X: f(x)=$ $g(x)=x\}$ of common fixed points of $f$ and $g$. As we shall show, if $f$ and $g$ are continuous and $X$ is compact metric, $f$ and $g$ are compatible iff they commute on the set $\{x \in X: f(x)=g(x)\}$ of coincidence points of $f$ and $g$. The purpose of this note is to consider and to emulate the relative merits of compatibility and commutativity of maps in the setting of compact metric spaces. We shall do so by proving three fixed point theorems which extend results by Fisher, Leader, Das and Debata, and the author.

As to notation, we let $R$ denote the reals with usual topology, $N$ the set of natural numbers, and $N_{0}=N \cup\{0\}$. If $f: X \rightarrow X, C_{f}$ denotes the set of all maps $g: X \rightarrow X$ which commute with $f$, and we shall write $f x$ for $f(x)$ when convenient.

\section{Compatible maps.}

DEFINITION $2.1[12]$. Self-maps $f$ and $g$ of a metric space $(X, d)$ are compatible iff $\lim _{n} d\left(f g x_{n}, g f x_{n}\right)=0$ when $\left\{x_{n}\right\}$ is a sequence such that $\lim _{n} f x_{n}=$ $\lim _{n} g x_{n}=t$ for some $t$ in $X$.

Thus, if $d(f g x, g f x) \rightarrow 0$ as $d(f x, g x) \rightarrow 0$, then $f$ and $g$ are compatible. So if $f$ and $g$ commute they are obviously compatible. On the other hand, let $f x=5 x^{3}$ and $g x=2 x^{3}$ for $x$ in $R$. Then $|g x-f x|=3|x|^{3} \rightarrow 0$ iff $x \rightarrow 0$, and $|f g x-g f x|=$ $210|x|^{9} \rightarrow 0$ iff $x \rightarrow 0$. So $f$ and $g$ are compatible although they do not commute. In fact, $f$ and $g$ are not even weakly commutative. Sessa defined self-maps $f$ and $g$ of $(X, d)$ to be weakly commuting iff $d(f g x, g f x) \leq d(f x, g x)$ for $x$ in $X$. Clearly, commuting maps are weakly commuting and weakly commuting maps are compatible, but neither implication is reversible as examples in [18] and the above example (respectively) show.

Received by the editors July 13, 1987.

1980 Mathematics Subject Classification (1985 Revision). Primary 54H25; Secondary 54E45. 
We now prepare to simplify the criterion given in Definition 2.1. by citing Proposition 2.2(1) of [12] which states that if self-maps $f$ and $g$ of a metric space are compatible then $f g x=g f x$ when $f x=g x$. An example to follow shows that the converse is not true in general. But upon noting that a mapping $f: X \rightarrow Y$ between topological spaces is proper iff $f^{-1}(C)$ is compact in $X$ when $C$ is compact in $Y$, we can say:

THEOREM 2.2. Let $f$ and $g$ be continuous self-maps of a metric space $(X, d)$. If $f$ is a proper map, then $f$ and $g$ are compatible iff $f x=g x$ implies $f g x=g f x$.

PROOF. The necessity of the condition follows from Proposition 2.2(1) of [12], or can be easily proved by supposing that $f(x)=g(x)$ and considering the sequence $\left\{x_{n}\right\}$ where $x_{n}=x$ for $n \in N$. To prove sufficiency let $\left\{x_{n}\right\}$ be a sequence in $X$ and suppose that

$$
\lim _{n} f x_{n}=\lim _{n} g x_{n}=t, \quad \text { for some } t \in X .
$$

Then $S=\left\{f x_{n}: n \in N\right\} \cup\{t\}$ is compact, so that $f^{-1}(S)$ is compact since $f$ is proper. Consequently, $\left\{x_{n}\right\}$ has a subsequence $\left\{x_{k_{n}}\right\}$ which converges to an element $c$ of $X$. Since $f$ and $g$ are continuous, $f x_{k_{n}} \rightarrow f c$ and $g x_{k_{n}} \rightarrow g c$. But then (1) implies

$$
f x_{n}, g x_{n} \rightarrow t=f(c)=g(c),
$$

and $f g c=g f c$ by hypothesis. Therefore, since $f g x_{n} \rightarrow f g c$ and $g f x_{n} \rightarrow g f c$ by (2) and the continuity of $f$ and $g, d\left(f g x_{n}, g f x_{n}\right) \rightarrow 0$ as desired.

EXAMPLE 2.4. Let $X=R, g(x)=2-x^{2}$ and $f(x)=x^{2} . f$ and $g$ are both continuous and proper. If $f(x)=g(x)$, then $x= \pm 1 . g f( \pm 1)=1=f g( \pm 1)$ so that $f$ and $g$ are compatible; but they are not weakly commuting (let $x=3$ ).

Since continuous self-maps of compact metric spaces are very proper, we have the following.

COROLlARY 2.3. Two continuous self-maps of a compact metric space are compatible iff they commute on their set of coincidence points.

The following example, referred to above, demonstrates the essential role played by "proper maps" in ensuring compatibility.

EXAMPLE 2.5. Let $f, g:[0, \infty) \rightarrow[0, \infty)$ be defined by $f(x)=x(x+1)^{-1}$ and $g(x)=(\underline{x}$ for $x<1$ and $\underline{1}$ for $x \geq 1)$. Now $f$ and $g$ are both continuous but neither is proper. Also, $f x=g x$ implies $x=0$, and $f(0)=g(0)=0=f g(0)=g f(0)$, so that the conditions of Theorem (2.2) except for that of being proper are met. But if $x_{n}=n$ for $n \in N$, then $\lim _{n} f x_{n}=\lim _{n} g x_{n}=1$, whereas $\lim _{n} d\left(f g x_{n}, g f x_{n}\right)=$ $d(1 / 2,1) \neq 0$; thus $f$ and $g$ are not compatible.

COROLLARY 2.6. Suppose that $f$ and $g$ are continuous self-maps of a metric space and that $f$ is proper. If $f x=g x$ implies $x=f x$, then $f$ and $g$ are compatible.

PROOF. If $f x=g x$, then $x=f x=g x$ and hence $f g x=g f x . f$ and $g$ are therefore compatible by Theorem 2.2 .

Note that the condition of Corollary 2.6 is sufficient but not necessary since the functions $f$ and $g$ of Example 2.4 are continuous, proper, and compatible, but $f(-1)=g(-1)=1$

The next result tells us that nice functions on nice spaces are compatible with lots of functions. 
COROLLARY 2.7. Let $M$ be a convex subset of a normed linear space and let $f: M \rightarrow M$ be proper and continuous. If $s: M \rightarrow[0,1)$ is continuous and if $g_{s}(x)=(1-s(x)) x+s(x) f(x)$ for $x$ in $M$, then $f$ and $g_{s}$ are compatible.

Proof. Since $M$ is convex, $g_{s}: M \rightarrow M$, and $g_{s}$ is continuous since $s$ and $f$ are. Moreover, if $g_{s}(x)=f(x)$, then $(1-s(x)) f(x)=(1-s(x)) x$ so that $f(x)=x$ since $s(x) \neq 1$; i.e., $f$ and $g_{s}$ are compatible by Corollary 2.6.

Functions of the form $g_{s}$ with $s$ constant give rise to iteration processes which produce sequences converging to fixed points of $f$ (see e.g., [3]). We refer the reader to [12] for further properties of compatible maps and for other examples which show that compatible maps need not be weakly commuting (and hence not commutative). Note also that Sessa has extended a variety of fixed theorems by substituting weak commutativity for commutativity; we cite [18 and 19] as examples.

3. A fixed point theorem for compatible maps. We appeal to the following generalization of a theorem of S. P. and S. L. Singh [20] to prove our next result.

THEOREM 3.1 [13]. Let $A, B, S$ and $T$ be self-maps of a complete metric space $(X, d)$. Suppose that $S$ and $T$ are continuous, the pairs $A, S$, and $B, T$ are compatible pairs, and that $A(X) \subset T(X)$ and $B(X) \subset S(X)$. If there exists $r \in(0,1)$ such that $d(A x, B y) \leq r \max (M x y)$ for $x, y$ in $X$, where

$$
M x y=\left\{d(S x, T y), d(A x, S x), d(B y, T y), \frac{1}{2}(d(A x, T y)+d(S x, B y))\right\},
$$

then there is a unique point $z$ in $X$ such that $A z=B z=S z=T z=z$.

The following theorem generalizes Theorem 1 of Fisher [7] by requiring compatibility in lieu of commutativity and replacing the terms $\frac{1}{2} d(A x, T y), \frac{1}{2} d(S x, B y)$ by $\frac{1}{2}(d(A x, T y)+d(S x, B y))$ in $M x y$.

THEOREM 3.2. Let $A, B, S, T$ be continuous self-maps of a compact metric space $(X, d)$ with $A(X) \subset T(X)$ and $B(X) \subset S(X)$. If $A, S$ and $B, T$ are compatible pairs and $d(A x, B y)<\max (M x y)$ (see (1)) when $\max (M x y)>0$, then $A, B, S$, and $T$ have a unique common fixed point.

PROOF. We assert $\max (M x y)=0$ for some pair $x, y$. Otherwise, the function $h x y=d(A x, B y) / \max (M x y)$ is continuous and satisfies $h x y<1$ on $X \times X$. Since $X \times X$ is compact, there exist $c, d \in X$ such that $h x y \leq r=h c d<1$ for $x, y \in X$. Consequently, $d(A x, B y) \leq r \max (M x y)$ on $X$ with $r<1$, so by Theorem 3.1, $A z=B z=S z=T z=z$ for some $z$. We have the contradiction, $\max (M z z)=0$ and $\max (M z z)>0$.

Since $\max (M x y)=0$ for some $x, y \in X,(1)$ implies

$$
S x=A x=T y=B y \text { and thus } S B y=S A x \text { and } A S x=A B y .
$$

Since $A$ and $S$ are compatible, $A x=S x$ in (2) implies that $S A x=A S x$ and therefore $S B y=A B y$. We now prove that $S B y=B y$ so that $B y$ is a common fixed point of $A$ and $S$.

For if $S B y \neq B y, \max (M B y y)>0$ by (1), so that by hypothesis

$$
\begin{aligned}
d(A B y, B y)<\max \{d(S B y, T y), d(S B y, A B y), & d(B y, T y), \\
& \left.\frac{1}{2}(d(A B y, T y)+d(S B y, B y))\right\} .
\end{aligned}
$$


Then (2) and the fact that $S B y=A B y$ imply

$$
d(A B y, B y)<\max \left\{d(A B y, B y), 0,0, \frac{1}{2}(d(A B y, B y)+d(A B y, B y))\right\},
$$

which yields the contradiction: $d(A B y, B y)<d(A B y, B y)$.

We thus have $A v=S v=v$, with $v=B y$. Similarly, there exists $w \in X$ such that $B w=T w=w$. Moreover, $v=w$. If not, (1) and the hypothesis imply

$$
d(v, w)<\max \left\{d(v, w), 0,0, \frac{1}{2}(d(v, w)+d(v, w))\right\}=d(v, w)
$$

again, a contradiction. We conclude that $v=w$ is the common fixed point of $A, B, S$, and $T$. In like manner, "uniqueness" follows immediately.

The following example verifies that Theorem 3.2 does indeed generalize Theorem 1 of Fisher referred to above.

EXAMPLE 3.3. Let $X=[0,1]$ and $d(x, y)=|x-y|$. Define $S x=x^{1 / 2}$, $T x=x^{1 / 2} / 2, A x=x^{1 / 2} / 4$, and $B x=x^{1 / 2} / 8 . A, B, S$, and $T$ are continuous, and $A(X)=\left[0, \frac{1}{4}\right] \subset\left[0, \frac{1}{2}\right]=T(X)$; similarly, $B(X) \subset S(X) . A$ and $S$ are compatible by Corollary 2.3 since $X$ is compact and $A x=S x$ implies $x=0=A S(0)=$ $S A(0)$. Likewise, $B$ and $T$ are compatible. Moreover, $d(A x, B y)=\frac{1}{4}(S x, T y) \leq$ $\frac{1}{4} \max (M x y)$, and the hypothesis of Theorem 3.2 is satisfied. However, $A$ and $S$ are not weakly commutative-and hence not commutative-since $|A x-S x|=$ $3 x^{1 / 4}|S A x-A S x|$, so that $|A x-S x|<|S A x-A S x|$ if $x<1 / 81$. The hypothesis of Fisher's Theorem 1 is therefore not satisfied.

To better appreciate how "tight" the hypothesis of Theorem 3.2 might be and to better understand the relative roles of commutativity and compatibility, consider the following.

THEOREM 3.4 (FISHER [6]). Let $A, B, S$, and $T$ be mappings of a compact metric space $(X, d)$ into itself satisfying

$$
d(A x, B y)<\max \{d(S x, T y), d(S x, A x), d(T y, B y), d(S x, B y), d(T y, A x)\}
$$

for all $x, y$ in $X$ for which the right-hand side of (3) is positive. If $A$ and $B$ commute, if $S$ and $T$ commute with $A B$ and if $A B$ is continuous, then $A, B, S$, and $T$ have a unique common fixed point.

Example 6 by Sessa [19] shows that Theorem 3.4 is false even if the only change in the hypothesis is to permit $S$ and $A B$ to be a weakly commuting pair.

4. Fixed point theorems for commuting maps. The proofs of our two remaining theorems appeal to the following.

PROPOSITION 4.1. Let $f$ and $g$ be commuting self-maps of a compact metric space $(X, d)$ such that $g f$ is continuous. If $A=\bigcap_{n=1}^{\infty}(g f)^{n}(X)$, then

(i) $h(A) \subset A$ for $h \in C_{g f}$,

(ii) $A=f(A)=g(A) \neq \varnothing$, and

(iii) $A$ is compact.

Proof. It is well known (see e.g., [14]) that $A$ is not empty, that $A$ is compact, and that $g f(A)=A$. If $h \in C_{g f}$, we can write

$$
h(A) \subset \bigcap_{n=1}^{\infty} h(g f)^{n}(X)=\bigcap_{n=1}^{\infty}(g f)^{n}(h(X)) \subset \bigcap_{n=1}^{\infty}(g f)^{n}(X)=A .
$$


Specifically, $g(A) \subset A$ and $f(A) \subset A$. Thus $A=g f(A) \subset g(A) \subset A$, so $g(A)=A$. Similarly, $f(A)=A$.

In our next result we use the standard notation $\operatorname{diam}(S)=\sup \{d(x, y): x, y \in S\}$ if $S$ is a subset of a metric space $(X, d)$.

THEOREM 4.2. Let $f$ and $g$ be commuting self-maps of a compact metric space $(X, d)$ such that $g f$ is continuous. If

(1) $f x \neq g y$ implies $d(f x, g y)<\operatorname{diam}\left\{h(z): z \in\{x, y\}\right.$ and $\left.h \in C_{g f}\right\}$, then there is a unique point $a$ in $X$ such that $a=f a=g a$. In fact, $a=h a$ for all $h \in C_{g f}$.

Proof. Let $A$ be as in Proposition 4.1 so that (i), (ii), and (iii) of Proposition 4.1 hold. We assert that $A=\{a\}$ for some $a$ in $X$. Otherwise $\operatorname{diam}(A)>0$, so by compactness there exist distinct $u, v \in A$ such that $d(u, v)=\operatorname{diam}(A)$. By (ii), we can find $x, y \in A$ such that $u=f x$ and $v=g y$; i.e., $d(f x, g y)=\operatorname{diam}(A)$. Since $f x \neq g y,(1)$ implies

$$
\operatorname{diam}(A)=d(f x, g y)<d\left(h_{1} z_{1}, h_{2} z_{2}\right)
$$

for some $h_{i} \in C_{g f}$ and $z_{i} \in\{x, y\}(i=1,2)$. Since $z_{i} \in A$, (i) of Proposition 4.1 implies that $h_{i} z_{i} \in A$ for $i=1,2$; consequently, (2) yields the contradiction, $\operatorname{diam}(A)<\operatorname{diam}(A)$.

Thus $A=\{a\}$ for some $a$ in $X$. Then (i) implies that $a=h a$ for $h$ in $C_{g f}$; in particular, $a=f a=g a$. Now if $c=f c=g c, g f c=c$ and thus $(g f)^{n} c=c$ for $n \in N$; i.e., $c \in A=\{a\}$. Hence, $a$ is the only common fixed point of $f$ and $g$.

Clearly, Theorem 3.4 follows from Theorem 4.2. For suppose that $A x \neq B y$. Then the right member of (3) in Theorem 3.4 is positive and therefore the inequality (3) holds. But since $S, T \in C_{A B}$ by hypothesis, (1) of Theorem 4.2 with $A=f$ and $B=g$ holds.

Observe also that since $A$ in the above proof is shown to be a singleton, Leader's Theorem $1\left(1^{\circ}\right.$ and $\left.7^{\circ}\right)$ in [14] assures us that $(g f)^{n}(x) \rightarrow a$ uniformly for all $x \in X$. Thus $a$ is a "uniformly contractive" point for $g f$, but it need not be for both $f$ and $g$ as examples show.

Note also that in light of the above comments, the theorem by Das and Debata [4] follows from Theorem 4.2, and the following corollary extends Corollary 2 of Leader [14].

COROLLARY 4.3. If $f$ is a continuous self-map of a compact metric space $(X, d)$ such that for some $r, s \in N_{0}$,

$$
f^{r} x \neq f^{s} y \text { implies } d\left(f^{r} x, f^{s} y\right)<\operatorname{diam}\left\{h z: z \in\{x, y\} \& h \in C_{f}\right\},
$$

then $f$ has a uniformly contractive fixed point.

PrOOF. By the proof of Theorem 4.2 with $f=f^{r}$ and $g=f^{s}, \bigcap_{n=1}^{\infty}\left(f^{r} f^{s}\right)^{n}(X)$ $=A=\{a\}$, a singleton. But $\bigcap_{n=1}^{\infty} f^{n}(X) \subset \bigcap_{n=1}^{\infty} f^{p n}(X)$ for any $p \in N$. Thus $\bigcap_{n=1}^{\infty} f^{n}(X)=\{a\}$, and the conclusion obtains by Leader's Theorem $1\left(1^{\circ}\right.$ and $7^{\circ}$ ).

Note. To appreciate the scope of Theorem 4.2 and hence of Corollary 4.3, observe that the functions $h \in C_{g f}$ in the right member of (1) include all functions of the form $F^{n}$ with $n \in N_{0}$ and $F=f, g, g f$, or any function in $C_{f} \cap C_{g}$. Our final result is a cousin to Theorem 4.2 , but "reverses" the inequality in (1). 
THEOREM 4.4. Let $f$ and $g$ be continuous commuting self-maps of a compact metric space $(X, d)$. If

$$
f x \neq g y \text { implies } d(h x, h y)<d(f x, g y) \text { for some } h \in C_{f} \cap C_{g},
$$

then at least one of $f$ or $g$ has a fixed point.

Proof. As above we let $A=\bigcap_{n=1}^{\infty}(g f)^{n}(X)$, so that (i)-(iii) of Proposition 4.1 hold, noting that $C_{f} \cap C_{g} \subset C_{g f}$. Since $f$ and $g$ are continuous and $A$ is compact, there exist, $a, b \in A$ such that

$$
d(a, f(a)) \leq d(x, f(x)) \quad \text { and } \quad d(b, g(b)) \leq d(x, g(x))
$$

for $x \in A$. We assume without loss of generality that

$$
d(a, f(a)) \leq d(b, g(b)) .
$$

Since $g(A)=A$ by (ii), $g(c)=a$ for some $c \in A$. But then, if $a \neq f(a), g(c) \neq$ $f(g(c))$, and (4) yields $h \in C_{f} \cap C_{g}$ such that $d(h(c), h(g(c)))<d(g(c), f(g(c)))=$ $d(a, f(a))$. Consequently,

$$
d(h(c), g(h(c)))<d(b, g(b))
$$

by (6) since $h \in C_{g}$. But since $h \in C_{f} \cap C_{g}$, (i) implies that $h(c) \in A$ and (7) therefore contradicts the right member of (5).

We conclude that the assumption $a \neq f(a)$ is false.

The following example reveals that not both $f$ and $g$ of Theorem 4.4 need have a fixed point and that the fixed point may not be unique.

EXAMPLE 4.4. Let $X=\{0,1\}, d(x, y)=|x-y|, h=g=i$-the identity map, and define $f$ by $f(0)=1, f(1)=0$. Then $f$ and $g$ are continuous commuting maps of a compact metric space into itself and $h \in C_{f} \cap G_{g}$. Moreover, $f(x) \neq g(y)$ implies $x=y$ or $i(x)=i(y)=h(x)=h(y)$, so $0=d(h(x), h(y))<d(f(x), g(y))$, and (4) holds. And $g$ has two fixed points while $f$ has none.

We conclude by observing that the sufficiency portion of Corollary 2.3 in [9] is a special case of Theorem 4.4 with $f=g$.

\section{REFERENCES}

1. Cheng Chun Chang, On a fixed point theorem of contractive type, Comment. Math. Univ. St. Paul 32 (1983), 15-19.

2. Shih-Sen Chang, $A$ common fixed point theorem for commuting mappings, Proc. Amer. Math. Soc. 83 (1981), 645-652.

3. C. E. Chidume, Iterative approximation of fixed points of Lipschitzian pseudo-contractive mappings, Proc. Amer. Math. Soc. 99 (1987), 283-288.

4. G. Das and J. P. Dabata, A note on fixed points of commuting mappings of contractive type, Indian J. Math. 27 (1985), 49-51.

5. K. M. Das and V. Naik, Common fixed point theorems for commuting maps on metric spaces, Proc. Amer. Math. Soc. 77 (1979), 269-373.

6. Brian Fisher, A common fixed point theorem for four mappings on a compact metric space, Bull Inst. Math. Acad. Sinica 12 (1984), 249-252.

7. $\_$, Common fixed points of four mappings, Bull. Inst. Math. Acad. Sinica 11 (1983), 103113.

8. Brian Fisher and M. S. Kahn, Some fixed point theorems for commuting mappings, Math. Nachr. 106 (1982), 323-326.

9. G. Jungck, Periodic and fixed points, and commuting mappings, Proc. Amer. Math. Soc. 76 (1979), 333-338. 
10. ___ Commuting mappings and fixed points, Amer. Math. Monthly 83 (1976), 261-263.

11. __ A common fixed point theorem for commuting maps on L-spaces, Math. Japon. 25 (1980), 81-85.

12. __ Compatible mappings and common fixed points, Internat. J. Math. Math. Sci. 9 (1986), 771-779.

13. _ Compatible mappings and common fixed points (2), Internat. J. Math. Math. Sci. 9 (1986), 285-288.

14. Solomon Leader, Uniformly contractive fixed points in compact metric space, Proc. Amer. Math. Soc. 86 (1982), 153-158.

15. R. D. Pant, Common fixed points of two pairs of commuting mappings, Indian J. Pure Appl. Math. 17 (1986), 187-192.

16. Sehie Park and Jong Sook Bae, Extensions of a fixed point theorem of Meir and Keeler, Ark. Math. 19 (1881), 223-228.

17. Barada K. Ray, Remarks on a fixed point theorem of Gerald Jungck, J. Univ. Kuwait Sci. 12 (1985), 169-171.

18. Salvatore Sessa, On a weak commutativity condition of mappings in fixed point considerations, Publ. Inst. Math. (Beograd) (N.S.) 32(46) (1982), 149-153.

19. __ On fixed points of weakly commuting mappings in compact metric spaces, Jñānābha 15 (1985), 79-91.

20. S. L. Singh and S. P. Singh, A fixed point theorem, Indian J. Pure Appl. Math. 11 (1980), 1584-1586.

21. S. L. Singh and B. M. L. Tivari, A note on recent generalizations of Jungck contraction principle, J. UPGC Acad. Soc. 3(1)(1986), 13-18.

Department of Mathematics, Bradley University, PeORia, Illinois 61625 